Supporting information for:

\title{
Mutation of $\beta$ GIn114 to Ala Alters the Stabilities of Allosteric States in Tryptophan Synthase Catalysis
}

Rittik K. Ghosh ${ }^{\dagger}$, Eduardo Hilario ${ }^{\S}$, Viktoriia Liu ${ }^{\S}$, Yangyang Wang ${ }^{\S}$, Dimitri Niks ${ }^{\dagger}$, Jacob B. Holmes $^{\S}$, Varun V. Sakhrani ${ }^{\S}$, Leonard J. Mueller ${ }^{\S, *}$, and Michael F. Dunn ${ }^{\dagger, *}$.

${ }^{\dagger}$ Department of Biochemistry, University of California, Riverside, California, 92521

$\S$ Department of Chemistry, University of California, Riverside, California, 92521

* Corresponding Authors (to whom correspondence should be addressed):

Leonard J. Mueller, Email: leonard.mueller@ucr.edu

Michael F. Dunn, Email: dunn@ucr.edu. 


\section{Additional Experimental Methods:}

Site-Directed Mutagenesis. The translational coupling gene encoding the $\alpha$ - and $\beta$-chains of WT TS was previously cloned into NcoI and SacI restriction sites to generate the construct pEBA-10 expression vector ${ }^{1}$. This construct was used as the DNA template during two-steps of polymerase chain reaction (PCR) to introduce specific point-mutations alongside the $\beta$-chain polynucleotide sequence of wild-type TS. Oligonucleotide TS-NcoI-FW and TS-SacI-REV, respectively, anneals upstream and downstream of the WT TS polynucleotide sequence in the pEBA-10 expression vector ${ }^{1}$. The first round of PCR reactions was performed using the pairs of nucleotide primers TS-NcoI-FW (5'- CAA TTT CAC ACA GGA AAC AGA CCA TGG -3') and

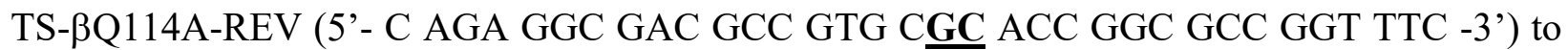
generate fragment $\mathrm{A}$, and TS- $\beta \mathrm{Q} 114 \mathrm{~A}-\mathrm{FW}$ (5'- GAA ACC GGC GCC GGT GCG CAC GGC

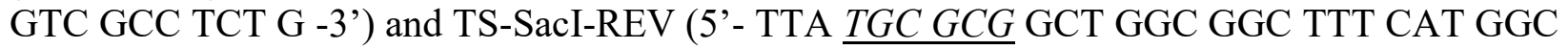
TGA G -3 ') to generate fragment $\mathrm{B}$, where an underlined and italic sequence corresponds to a restriction site, and an underlined and bold sequence corresponds to a mutation. The purification of PCR products A and B from agarose gel slices was carried out by using Ultra-Sep gel extraction kit (Omega Bio-Tek). Fragments A and B were mixed in equimolar amounts, heat denatured, annealed, and the recombinant strands were extended. Then, oligo primers TS-NcoIFW and TS-SacI-REV were added, and a second PCR was performed. The fragment encoding the $\beta$ Q114A TS mutant was digested with NcoI and SacI, gel purified, and ligated with pEBA-10 vector, previously digested with the same enzymes and gel purified ${ }^{2}$. The recombinant plasmid construct $\beta$ Q114A TS was DNA sequenced to confirm the polynucleotide sequence. All molecular biology steps were followed as described in Molecular Cloning: A Laboratory Manual $^{3}$.

Enzymes. The wild-type $\alpha_{2} \beta_{2}$ TS was purified as previously described ${ }^{4,5}$. The $\beta$ Q $114 \mathrm{~A}$ mutant enzyme was purified by a modification of the procedure used for wild-type $\alpha_{2} \beta_{2}$. Cells containing the mutant plasmid were grown in LB media with IPTG induction. The cell extracts were dissolved in Tris-HCl buffer and then adjusted to 45\% Ammonium Sulfate saturation by stepwise addition. The yellow pellet was re-dissolved in a minimal volume of buffer and applied to a Sephadex S-200 gel filtration column $(60 \times 2.5 \mathrm{~cm})$, eluting at $1 \mathrm{~mL} / \mathrm{min}$. The yellow fractions containing $\beta$ Q114A TS were pooled and concentrated in a centrifugal concentrator (Vivaspin, Sartorius) with a $30000 \mathrm{MW}$ cutoff. An extra dialysis step in Bicine buffer containing EDTA and Cesium Chloride $(\mathrm{CsCl}), \mathrm{pH} 7.8$, after size exclusion column separation, was added to ensure $\mathrm{Cs}^{+}$ion incorporation in the enzyme. Enzyme active site concentrations were estimated from the UV/Vis absorbance at $412 \mathrm{~nm}$ based on $\varepsilon_{412}=7.3 \mathrm{mM}^{-1} \mathrm{~cm}^{-1}$. Yields of both WT and $\beta Q 114 \mathrm{~A}$ TS were $>100 \mathrm{mg} / \mathrm{L}$ of culture.

Crystallization Assays. TS samples were centrifuged at 10,000 g for $10 \mathrm{~min}$ to clarify the solution before initiating any crystallization trials. Equal volumes $(4 \mu \mathrm{L})$ of protein solution $(15$ $\mathrm{mg} \mathrm{ml}^{-1}$ ) and reservoir solution were mixed and equilibrated against a $500 \mu \mathrm{L}$ reservoir solution consisting of $50 \mathrm{mM}$ bicine-CsOH, pH 7.8, $50 \mathrm{mM} \mathrm{CsCl,} \mathrm{6-10 \%} \mathrm{PEG} \mathrm{8000,} \mathrm{2-8} \mathrm{mM} \mathrm{spermine,} 1$ $\mathrm{mM}$ 2-(\{[4-(trifluoromethoxy)phenyl]sulfonyl $\}$ amino)ethyl dihydrogen phosphate (PDB ID = F9F) ${ }^{6}$. The crystal quality was optimized by screening different concentrations of PEG 8000 (6$10 \%)$ and spermine $(2-8 \mathrm{mM})$ in the reservoir solution. Crystals of tryptophan synthase were grown using a 24-well sitting-drop vapor-diffusion plate (Cryschem M plate, HR1-002 
(Hampton Research) at $25^{\circ} \mathrm{C}$. Large TS crystals $(80-200 \mu \mathrm{m})$ were used for X-ray diffraction experiments.

X-ray Data Collection and Analysis. Prior to X-ray diffraction data collection, crystals were briefly immersed in cryoprotectant consisting of the reservoir solution containing $30 \%$ dimethyl sulfoxide (DMSO) and mounted in a nylon-fiber loop (Hampton Research, USA). Other TS $\beta Q 114 \mathrm{~A}$ crystals were soaked in cryoprotectant buffer containing specific compounds such as LSerine, and 2-aminophenol (PDB ID: 2AF), F9 (PDB ID: F9F). Crystals were flash-frozen in liquid nitrogen and shipped to the Northeastern Collaborative Access Team beamline 24ID-C at the Advanced Photon Source (APS), Argonne National Laboratory, for X-ray diffraction experiments. All X-ray diffraction data were collected at a wavelength of $0.9792 \AA$ with a pixel array detector Pilatus-6MF. X-ray diffraction intensities were processed using iMOSFLM ${ }^{7}$ and them scaled with Scala ${ }^{8}$, implemented in the CCP4 package ${ }^{9}$. The molecular replacement step was performed in Phaser ${ }^{10}$ using PDB ID: 4HT3 ${ }^{4}$ as search model.

Microcrystalline Protein Samples for Solid-State NMR. Microcrystalline samples of $\beta Q 114 \mathrm{~A}$ TS were prepared by diluting the enzyme solution 1:1 with $50 \mathrm{mM} \mathrm{Cs}^{+}$bicine buffer, $\mathrm{pH} 7.8$, containing 20\% PEG-8000 and $2.0 \mathrm{mM}$ spermine as previously described ${ }^{11}$. Yellow colored microcrystals were collected and washed with $50 \mathrm{mM} \mathrm{Cs}^{+}$-bicine buffer, $\mathrm{pH} 7.8$, containing $10 \%$ PEG-8000, $1 \mathrm{mM}$ spermine, and $2 \mathrm{mM} \mathrm{F9}$. The crystals were packed at $10000 \mathrm{~g}$ into a Bruker 4 $\mathrm{mm}$ magic-angle spinning (MAS) rotor with an approximate volume of $50 \mu \mathrm{L}$; each rotor contained $60-70 \mathrm{mg}$ of protein. For making the $\mathrm{E}\left(\mathrm{C}_{3}\right)_{2 \mathrm{AP}}$ carbanion, L-Ser was introduced by direct addition of 3-5 $\mu \mathrm{L}$ of 1.2 M L-Ser to the packed MAS rotor, while 2AP was introduced by addition of $4 \mu \mathrm{L}$ of a concentrated stock solution of $2 \mathrm{AP}(\sim 250 \mathrm{mM})$ in acetonitrile.

X-ray Structure Determination. The water content varies considerably in TS structures. In this work, the Matthews coefficient ${ }^{12}$ with units of $\AA^{3} / \mathrm{Da}$ and the water content are $2.60(52.64 \%$ water content), $2.50(50.83 \%), 2.64(53.38 \%), 2.63(53.30 \%)$ and $2.52(51.24 \%)$ for crystal structures $6 \mathrm{DZ} 4,6 \mathrm{DZO}, 6 \mathrm{D} 0 \mathrm{~V}, 601 \mathrm{H}$ and $6 \mathrm{C} 73$ respectively. These values fall within the range typically observed for $70 \mathrm{kDa}$ crystal structures ${ }^{13}$. During refinement, the $2 \mathrm{Fo}-\mathrm{Fc}$ map was kept at counter level cut off of $1.00 \sigma$ during manual modeling and fitting. The crystals were prepared in buffer containing either sodium chloride or cesium chloride. $\mathrm{Na}^{+}$and $\mathrm{Cs}^{+}$bind to a welldescribed monovalent cation binding site within the $\beta$-subunit of TS and cation binding to this site is important for the biological functioning of $\mathrm{TS}^{14}$. At the $\beta$ subunit of $\mathrm{TS}$, there is a monovalent cation site. Based on the structure factors and B-factor analysis, the electron densities at this site were easily distinguished from water, ammonium ion or chloride ion in the reported structures. The average water B-factor for each of the new crystal structures 6DZ4, $6 \mathrm{DZO}, 6 \mathrm{D} 0 \mathrm{~V}, 601 \mathrm{H}$ and $6 \mathrm{C} 73$ respectively are $38.8,32.3,35.5,45.1,32.5$ and $34.5 \AA^{2}$. While modelling the electron density maps, care was taken to avoid interpreting noise as water molecules at the reported resolution.

Based on the validation report and refinement statistics as is shown in Table 1 , the $0.2 \%$ disallowed residues for the Ramachandran plot belong to the residue $\alpha \mathrm{Phe} 212$. This is due to the presence of the $\alpha$-site ligand F9, which disturbs the phi/psi angles of the $\alpha$ Phe212. To our best of knowledge, all the crystal structures with F9 bound to the $\alpha$-site reported in the pdb database have this feature in common. 


\section{Conformational States in StTS $\beta$-subunit and PfTrpB: Comparison of the COMM Domain Positions}

A careful alignment of the StTS crystal structures 4HT3 (E(Ain)), 6DZ4 (E(Aex 1$)$ ), and 4HN4 (E(A-A)) in Chimera ${ }^{17}$ shows that except for small changes introduced by substrate bonding with the PLP cofactor (see Figure S1) there is no significant displacement of the COMM domain when $\mathrm{E}(\mathrm{Ain})$ is converted to $\mathrm{E}\left(\mathrm{Aex}_{1}\right)$; both conformations are designated as open $(\mathrm{T})$. However, upon formation of the $\alpha$-aminoacrylate, there is a sizable conformational change, with a displacement of the COMM domain of $\sim 3.0 \AA$ as the subunit adopts the closed (R) conformation. Analysis of the engineered PfTrpB structures 5DVZ (E(Ain)) and 5DW0 $\left(\mathrm{E}\left(\mathrm{Aex}_{1}\right)\right)$ shows there is a significant displacement of the COMM domain $(\sim 2.0 \AA)$ in the chemical transformation of $\mathrm{E}(\mathrm{Ain})$ to $\mathrm{E}\left(\mathrm{Aex}_{1}\right)$, with the latter designated as a partially closed (R') structure ${ }^{15,16}$. The interconversion of Pf $\operatorname{TrpB} \mathrm{E}\left(\mathrm{Aex}_{1}\right)$ to E(A-A) (PDB ID: 5VM5) results in the formation of a closed structure that is consistent with the closed $\beta$-subunit structure in StTS.

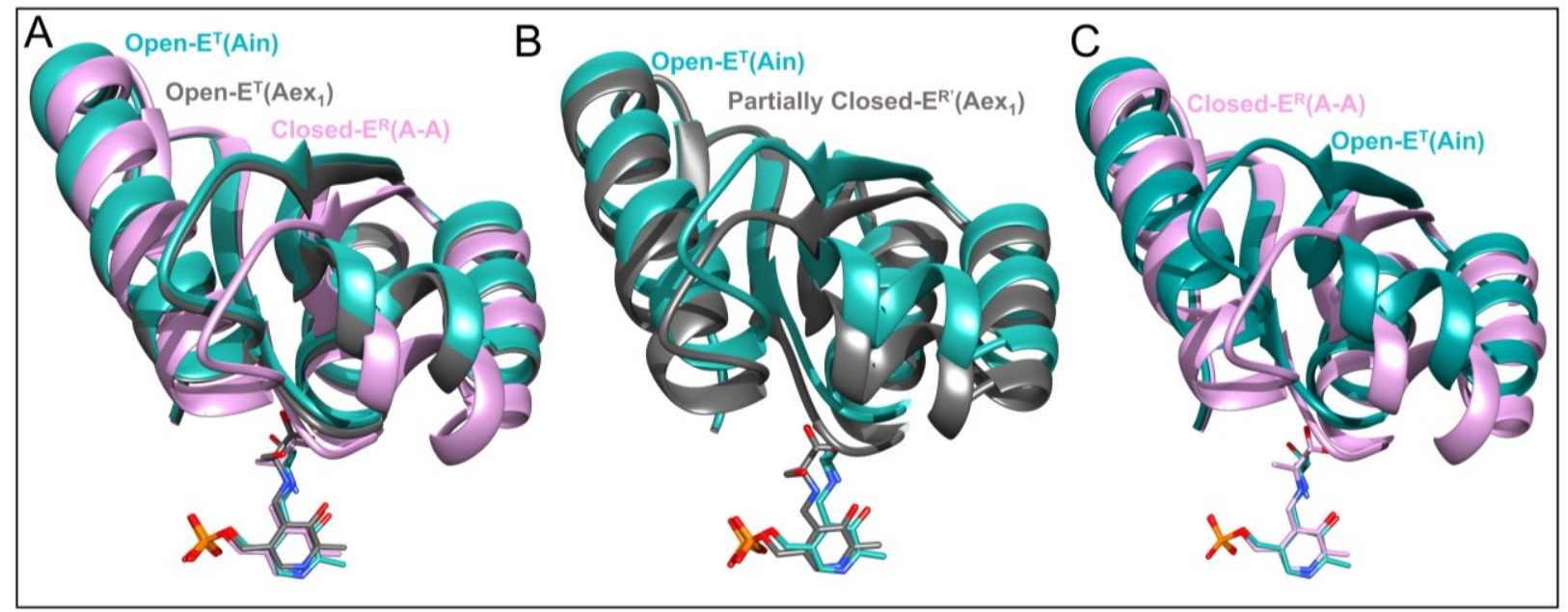

Figure S1: Comparisons of the $\beta$-subunit COMM domain and PLP cofactor positions for aligned crystal structures of $S t \mathrm{TS}$ and PfTrpB. (A) SfTS: Open (T) complexes $E^{\mathrm{T}}$ (Ain) (teal) and $\mathrm{E}^{\mathrm{T}}\left(\mathrm{Aex}_{1}\right)$ (grey), and the closed (R) complex $\mathrm{E}^{\mathrm{R}}(\mathrm{A}-\mathrm{A})$ (pink). (B) PfTrpB: Open (T) complex $\mathrm{E}^{\mathrm{T}}$ (Ain) (teal) and partially closed (R') complex $\mathrm{E}^{\mathrm{R}^{\prime}}\left(\mathrm{Aex}_{1}\right)$ (grey). (C) PfTrpB: Open (T) complex $\mathrm{E}^{\mathrm{T}}$ (Ain) (teal) and closed (R) complex $\mathrm{E}^{\mathrm{R}}(\mathrm{A}-\mathrm{A})$ (pink). Coloring Scheme: Ribbons and carbons are colored as: teal-blue in the $E\left(\right.$ Ain) structures, dark grey in the $E\left(A e x_{1}\right)$ structures, and pink in E(A-A) structures. Stick structures: Oxygen, nitrogen, and phosphorous atoms are colored red, blue and orange respectively and carbons retain the colors of the corresponding ribbons. Images rendered in Chimera ${ }^{17}$. 


\section{Computational Modelling of a Model Gem-Diamine Intermediate:}

Preparation of the gem-diamine model compound. A model for the E(GD) intermediate was constructed and geometry optimized in Gaussian09 ${ }^{18}$ using DFT (B3YLP; 6-311G) (Figure $\mathrm{S} 2 \mathrm{~B}$ ). The UV/Vis spectrum of the optimized structure was calculated using TDDFT (Figure $\mathrm{S} 2 \mathrm{~A}$ ) as discussed in the materials and methods section.

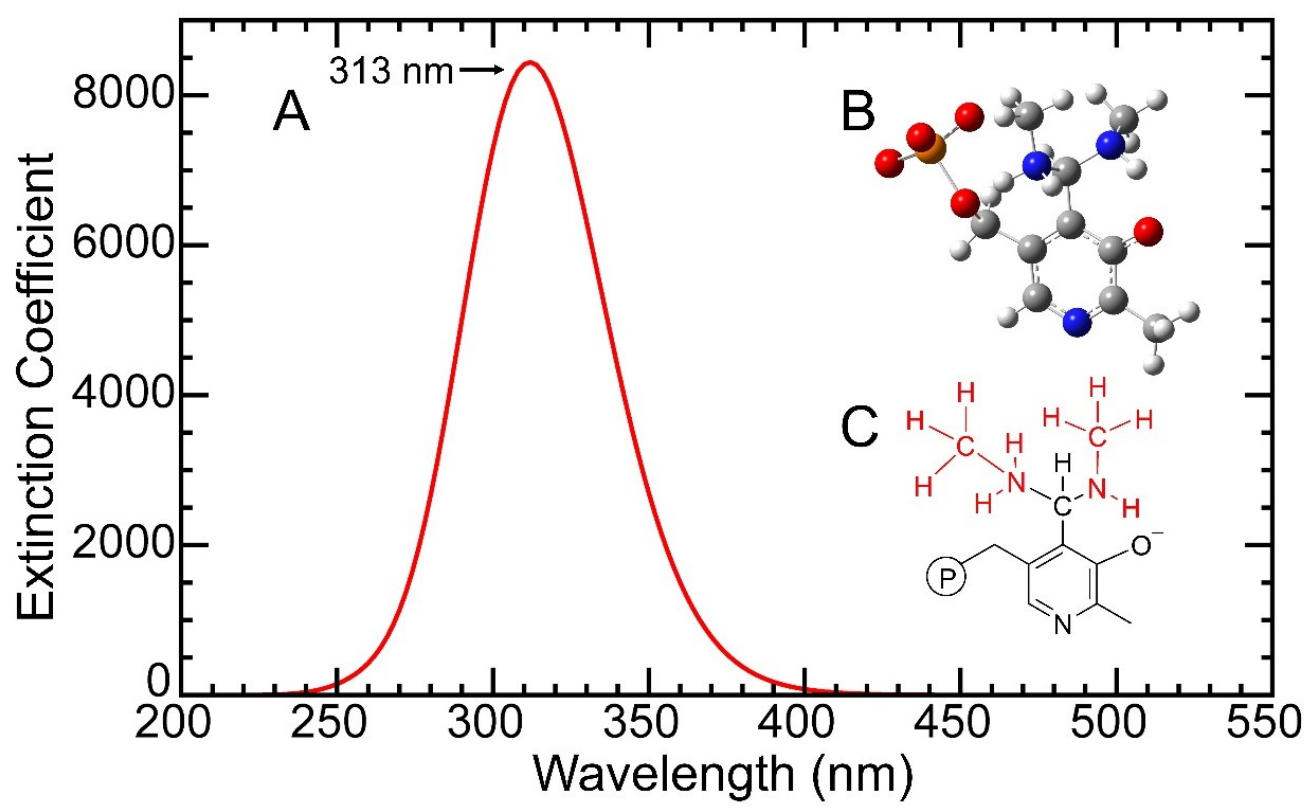

Figure S2: (A) Simulated UV/Vis spectrum of a model gem-diamine complex from TDDFT calculations. Figure rendered in Mathematica 12.1 ${ }^{19,20}$. (B) Model gem-diamine complex in ball and stick representation. (C) Scheme of the model gem-diamine used in the calculation.

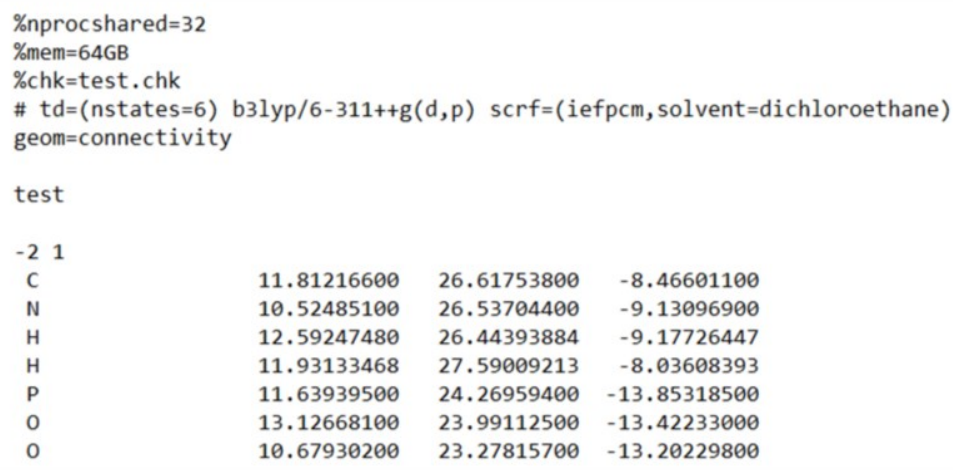

Figure S3: A snippet of the Gaussian input file. 


\section{References}

(1) Ahmed, S. A., Miles, E. W., and Davies, D. R. (1985) Crystallization and preliminary X-ray crystallographic data of the tryptophan synthase alpha $2 \beta 2$ complex from Salmonella typhimurium, J Biol Chem 260, 3716-3718.

(2) Hilario, E., Fan, L., Mueller, L. J., and Dunn, M. F. (2020) PCR Mutagenesis, Cloning, Expression, Fast Protein Purification Protocols and Crystallization of the Wild Type and Mutant Forms of Tryptophan Synthase, J Vis Exp 163.

(3) Sambrook, J., Fritsch, E. F., and Maniatis, T. (1989) Molecular cloning: a laboratory manual, Cold spring harbor laboratory.

(4) Niks, D., Hilario, E., Dierkers, A., Ngo, H., Borchardt, D., Neubauer, T. J., Fan, L., Mueller, L. J., and Dunn, M. F. (2013) Allostery and substrate channeling in the tryptophan synthase bienzyme complex: evidence for two subunit conformations and four quaternary states, Biochemistry 52, 6396-6411.

(5) Caulkins, B. G., Young, R. P., Kudla, R. A., Yang, C., Bittbauer, T. J., Bastin, B., Hilario, E., Fan, L., Marsella, M. J., Dunn, M. F., and Mueller, L. J. (2016) NMR Crystallography of a Carbanionic Intermediate in Tryptophan Synthase: Chemical Structure, Tautomerization, and Reaction Specificity, J Am Chem Soc 138, 15214-15226.

(6) Hilario, E., Caulkins, B. G., Huang, Y. M. M., You, W. L., Chang, C. E. A., Mueller, L. J., Dunn, M. F., and Fan, L. (2016) Visualizing the tunnel in tryptophan synthase with crystallography: Insights into a selective filter for accommodating indole and rejecting water, Bba-Proteins Proteom 1864, 268-279.

(7) Battye, T. G., Kontogiannis, L., Johnson, O., Powell, H. R., and Leslie, A. G. (2011) iMOSFLM: a new graphical interface for diffraction-image processing with MOSFLM, Acta Crystallogr D Biol Crystallogr 67, 271-281.

(8) Evans, P. R. (2011) An introduction to data reduction: space-group determination, scaling and intensity statistics, Acta Crystallogr D Biol Crystallogr 67, 282-292.

(9) Winn, M. D., Ballard, C. C., Cowtan, K. D., Dodson, E. J., Emsley, P., Evans, P. R., Keegan, R. M., Krissinel, E. B., Leslie, A. G., McCoy, A., McNicholas, S. J., Murshudov, G. N., Pannu, N. S., Potterton, E. A., Powell, H. R., Read, R. J., Vagin, A., and Wilson, K. S. (2011) Overview of the CCP4 suite and current developments, Acta Crystallogr D Biol Crystallogr 67, 235-242.

(10) Bunkoczi, G., Echols, N., McCoy, A. J., Oeffner, R. D., Adams, P. D., and Read, R. J. (2013) Phaser.MRage: automated molecular replacement, Acta Crystallogr D Biol Crystallogr 69, 2276-2286.

(11) Caulkins, B. G., Bastin, B., Yang, C., Neubauer, T. J., Young, R. P., Hilario, E., Huang, Y. M., Chang, C. E., Fan, L., Dunn, M. F., Marsella, M. J., and Mueller, L. J. (2014) Protonation states of the tryptophan synthase internal aldimine active site from solid-state NMR spectroscopy: direct observation of the protonated Schiff base linkage to pyridoxal5'-phosphate, J Am Chem Soc 136, 12824-12827.

(12) Matthews, B. W. (1968) Solvent content of protein crystals, J Mol Biol 33, 491-497.

(13) Kantardjieff, K. A., and Rupp, B. (2003) Matthews coefficient probabilities: Improved estimates for unit cell contents of proteins, DNA, and protein-nucleic acid complex crystals, Protein Sci 12, 1865-1871.

(14) Dierkers, A. T., Niks, D., Schlichting, I., and Dunn, M. F. (2009) Tryptophan synthase: structure and function of the monovalent cation site, Biochemistry 48, 10997-11010. 
(15) Buller, A. R., Brinkmann-Chen, S., Romney, D. K., Herger, M., Murciano-Calles, J., and Arnold, F. H. (2015) Directed evolution of the tryptophan synthase $\beta$-subunit for standalone function recapitulates allosteric activation, Proc Natl Acad Sci U S A 112, 1459914604.

(16) Maria-Solano, M. A., Iglesias-Fernandez, J., and Osuna, S. (2019) Deciphering the Allosterically Driven Conformational Ensemble in Tryptophan Synthase Evolution, $J$ Am Chem Soc 141, 13049-13056.

(17) Pettersen, E. F., Goddard, T. D., Huang, C. C., Couch, G. S., Greenblatt, D. M., Meng, E. C., and Ferrin, T. E. (2004) UCSF Chimera--a visualization system for exploratory research and analysis, J. Comput. Chem. 25, 1605-1612.

(18) Frisch, M., Trucks, G., Schlegel, H., Scuseria, G., Robb, M., Cheeseman, J., Scalmani, G., Barone, V., Mennucci, B., and Petersson, G. (2009) Gaussian 09, revision A. 02, vol 19. Gaussian, Inc., Wallingford, 227-238.

(19) Wolfram Research, I. (2020) Mathematica, 12.1 ed., Wolfram Research, Inc, Champaign, Illinois.

(20) Caprio, M. A. (2005) LevelScheme: A level scheme drawing and scientific figure preparation system for Mathematica, Comput Phys Commun 171, 107-118. 